Díaz, M., Llorente, M., y Méndez, P. (2015). Ciclo inmobiliario y demográfico. Un análisis de cointegración para el caso español. Revista Lebret, 7. Bucaramanga, Colombia: Universidad Santo Tomás, pp. 155 -174. ISSN: 2145-5996.

\title{
Ciclo inmobiliario y demográfico. Un análisis de cointegración para el caso español
}

\author{
Real estate and demographic cycle \\ A co-integration analysis for the spanish case
}

\author{
Montserrat Diaz Fernández ${ }^{l}$ \\ Mar Llorente Marron ${ }^{2}$ \\ Paz Méndez Rodríguez ${ }^{3}$
}

\begin{abstract}
Resumen
Se analiza la interrelación entre el ciclo inmobiliario y el comportamiento demográfico en España durante 1975 a 2013. Se evalúa empíricamente la existencia de un modelo de comportamiento entre ambos ciclos, siguiendo la metodología tradicional de los estudios de series de tiempo. Se especifica un modelo de corrección de error. Se evalúa el dominio del efecto de largo plazo sobre el corto plazo. El modelo obtenido detecta un equilibrio a largo plazo entre ambas series, ecuación de cointegración que visualiza el dominio de la serie inmobiliaria.
\end{abstract}

\section{Palabras clave}

Vivienda, alojamiento, natalidad, análisis de cointegración.

\section{Clasificación JEL: C32, J13, O15}

\begin{abstract}
The relationship between the real estate cycle and demographic behavior in Spain during the period 1975-2013 is analyzed. The existence of a pattern of behavior between the two cycles following the traditional methodology of time series studies are empirically evaluated. A model error correction is specified. The domain of the long-term effect on the short term is evaluated. The model detects a long-term equilibrium between the two series, a cointegrating equation that displays the domain of the real number estate series.
\end{abstract}

\section{Keywords}

Living place, housing, birth, cointegration analysis.

* Este artículo es derivado de la investigación "Modelización del mercado de la vivienda, liderado por el Departamento de Economía Cuantitativa de la Universidad de Oviedo. Este trabajo fue presentado en International Conference on Regional Science: Financing and the role of the regions and towns in economy recovery, 20 y 21 de 2014, Universidad de Zaragoza.

1 Doctorado en Ciencias Económicas y Empresariales. Docente del Departamento de Economía Cuantitativa, Facultad de Economía y Empresa, Universidad Oviedo. Correo electrónico: mdiaz@uniovi.es

2 Doctorado en Métodos Cuantitativos. Docente del Departamento de Economía Cuantitativa, Facultad de Economía y Empresa, Universidad Oviedo. Correo electrónico: mmarron@uniovi.es

3 Doctorado en Organización y Gestión de Empresas. Docente del Departamento de Economía Cuantitativa, Facultad de Economía y Empresa, Universidad Oviedo. Correo electrónico: mpmendez@uniovi.es 


\section{Introducción}

En los últimos años el protagonismo adquirido por el mercado de la vivienda resulta incuestionable. En España el ritmo de construcción ha sido muy intenso especialmente desde la segunda mitad de la década de los noventa habiéndose construido comparativamente más viviendas que en otros países del entorno europeo con mayor crecimiento demográfico, como Francia o Alemania. El precio de la vivienda aumentó significativamente en relación con la renta familiar, contribuyendo a la revalorización del patrimonio inmobiliario y a hacer del recurso a la financiación externa una cuestión insoslayable (Naredo, 2004).

Los efectos de la dinámica demográfica sobre el mercado residencial son obvios, la vivienda acompaña al efectivo poblacional en el transcurso de su ciclo de vida (Clark y Dieleman, 1996). El stock de vivienda influye en las oportunidades de crecimiento de la población a través de la inmigración y formación de nuevos hogares que a su vez condicionan la fecundidad (Feijten y Mulder, 2002).

La vivienda, bien necesario que satisface la condición de alojamiento, acompaña al efectivo poblacional a lo largo de su ciclo de vida. Desde esta perspectiva las decisiones de acceso a la misma se vinculan a intervalos temporales de largo plazo y complejas relaciones causales. El sector inmobiliario encuentra buena parte de la demanda de su producto en la dinámica demográfica, demanda a largo plazo, aunque dicha correspondencia no sea biunívoca. Estructura del hogar, proceso migratorio, envejecimiento, descenso de la fecundidad, emancipación juvenil, son aspectos de distinta naturaleza que integran la dinámica demográfica y están sujetos a una permanente interacción con la dinámica del mercado de la vivienda (Vinuesa, 2005). En el corto plazo las variables económicas, financieras y fiscales, además del alojamiento constituyen los factores determinantes de su demanda. La vivienda constituye un activo económico generador de riqueza.

Dentro del marco de las profundas transformaciones acaecidas en nuestra sociedad las que afectan a la evolución demográfica son sin duda relevantes. El descenso generalizado de los indicadores de fecundidad, aumento de la esperanza de vida, acentuado proceso de envejecimiento y desaceleración significativa del ritmo de crecimiento de la población constituyen los rasgos distintivos del contexto demográfico actual.

La evolución de la natalidad constituye un fenómeno determinante en el desarrollo demográfico español durante las últimas décadas. El fenómeno del babyboom no fue en España, como en otros países desarrollados, tan pronunciado ni tan temprano. Durante el período comprendido entre 1955 y 1975 la cifra de nacimientos superó los 650000 anuales. El descenso registrado de forma continua e intensa por los indicadores desde la segunda mitad de la década de los setenta del siglo XX ha hecho que España haya pasado de tener una de las tasas de natalidad más elevadas de Europa durante la década anterior a una situación diferente que condiciona además 
el desarrollo demográfico posterior. En la actualidad la generación conocida como baby-boomers representa casi un tercio de la población española, contribuyendo decisivamente a la dinámica del mercado inmobiliario.

En el análisis de la evolución demográfica y coyuntura económica, los cambios económicos que la preceden e inducen y el contexto demográfico que antecede, acompaña y provoca su desarrollo, estrechamente interrelacionados. La dinámica, evolución y comportamiento demográfico determina la cantidad y calidad de los servicios de vivienda demandada. El descenso de los indicadores de fecundidad generará una disminución del tamaño de las cohortes inferiores de la pirámide poblacional, población infantil a corto plazo y población joven a medio y largo plazo que repercutirá en el funcionamiento del mercado de la vivienda. En el mismo sentido el proceso de envejecimiento se dejará sentir también en la dinámica del mercado de la vivienda en cuya demanda el capital humano constituye un factor determinante (Eichholtz y Lindenthal, 2010).

La coyuntura socio-económica ha favorecido el comportamiento de la renta familiar y de las expectativas de los hogares (creación de empleo, aumento del número de ocupados por hogar, crecimiento del salario real) mejorando su capacidad adquisitiva. En la trayectoria de la natalidad la consideración de los hijos como un bien normal, aunque pudiera producirse una interacción cantidad-calidad, hace parte del análisis de la dinámica económica y se configura como una variable imprescindible (Becker, 1960). Actuaciones sobre las condiciones socioeconómicas que repercutan favorablemente sobre los indicadores de fecundidad trasladarán al mercado de la vivienda efectos estimuladores (Lim y Lee, 2013).

En el proceso de toma de decisión conocer el mecanismo de interrelación entre las variables demográficas y el sector inmobiliario es sin duda relevante. La identificación de la relación causal dirige metodológicamente el análisis a modelos teóricos diferentes, modelo clásico, neoclásico, nueva economía de la familia, entre otros (Climent y Meneu, 2003).

La interrelación entre las variables demográficas y la dinámica inmobiliaria constituye un aspecto ampliamente analizado en la literatura económica con manifestaciones diferentes temporal y territorialmente (Myers, 1990; Clark y Dieleman, 1996). La aproximación demográfica a través del crecimiento poblacional, proceso de envejecimiento o comportamiento de la fecundidad son algunos ejemplos para tener en cuenta. En la determinación de la demanda futura de necesidades de alojamiento el crecimiento poblacional juega un papel determinante (Vinuesa, 2003). El mercado inmobiliario recoge en cierta medida efectos dinamizadores como consecuencia de estímulos derivados de una coyuntura económica favorable sobre el comportamiento de la fecundidad. La conexión entre la dinámica demográfica y el sector inmobiliario también se dirige en la literatura económica a la presión que la primera pueda ejercer sobre la trayectoria del precio de la vivienda y los efectos, en consecuencia, negativos en relación con el comportamiento de la demanda de 
vivienda (Mankiw y Weil, 1989). No obstante, algunas críticas a las especificaciones de los modelos que analizan la interrelación descrita atenúan en parte dicha valoración (Hamilton, 1991; Green y Hendershott, 1995).

El objetivo de este trabajo consiste en analizar conjuntamente la trayectoria y relación existente entre la dinámica demográfica reciente y el mercado de la vivienda. Se evalúa un modelo de interrelación agregado entre el ciclo inmobiliario, VIVIENDAS y, el demográfico, NACIMIENTOS, en España que aporte evidencia empírica y visualice el carácter endógeno, unidireccional o bidireccional, o exógeno de ambos ciclos. Desde la óptica de la demanda, las variables demográficas ocupan indudablemente un papel destacado como indicador adelantado de la demanda futura (Bover, 1993). La dinámica del mercado de la vivienda afecta a la natalidad, a través del efecto renta y su consideración de activo financiero (Dettling y Kearney, 2014). La influencia directa e indirecta entre ambos ciclos se produce a través de la calidad de la vivienda y emancipación, convivencia y matrimonio, respectivamente (Ermisch, 1987; Mulder, 2006).

El uso de técnicas derivadas del análisis de cointegración permite profundizar positivamente en el análisis planteado, además de obtener una relación de equilibrio a largo plazo y resultados libres de correlaciones espúreas y parametrizaciones inestables.

\section{Material y método}

En el ámbito de la investigación económica la conexión del análisis de cointegración y Mecanismo de Corrección de Error (MCE) reconcilia posturas parcialmente divergentes. La metodología econométrica se fundamenta en el conocimiento de la realidad económica y en la capacidad de establecimiento de relaciones causales. El análisis de cointegración, siempre que las relaciones fundamentales entre las variables económicas estén correctamente especificadas y respondan a un interés real de mejora del conocimiento económico, constituye un perfeccionamiento de la cuestión (Guisán, 2002). La aproximación econométrica del fenómeno económico mediante las técnicas derivadas del análisis de cointegración permite obtener una relación de equilibrio a largo plazo y resultados libres de correlaciones espurias y parametrizaciones inestables. La posibilidad de complementar las relaciones de equilibrio de largo plazo de la ecuación de cointegración con la dinámica que incorpora el MCE enfatiza la significatividad de la metodología de cointegración.

Dos variables $x_{t}$ e $y_{t}$ integradas de primer orden, $I(1)$, están cointegradas cuando existe una combinación lineal de ambas, estacionaria de orden cero, $I(0)$, lo que implica la existencia de una relación de equilibrio a largo plazo. La cointegración entre dos variables I(1) equivale a analizar la estacionariedad del término de perturbación aleatoria del modelo que las relaciona. 
Los sistemas que incorporan en su especificación variables cointegradas, de acuerdo con el teorema de representación de Granger, pueden ser formulados como MCE que permiten modelizar las relaciones de largo y corto plazo.

$$
\Delta y_{t}=\gamma\left(\Delta x_{t}\right)+\alpha\left(y_{t-1}-\beta_{1}-\beta_{2} x_{t-1}\right)+\varepsilon_{t}
$$

donde, $\alpha\left(y_{t-1}-\beta_{1}-\beta_{z} x_{t-1}\right)$ denota el MCE siendo $\alpha<0$; el parámetro $\gamma$ mide el efecto a corto plazo que la variable $x_{t}$ ejerce sobre $y_{t} ; \beta_{2}$, recoge el efecto a largo plazo que la variable $x_{t}$ ejerce sobre $y_{t} \mathrm{y} \varepsilon_{t}$, el término de perturbación aleatoria. La formulación estacionaria de variables no estacionarias

$$
\Delta y_{t}=\gamma\left(\Delta x_{t}\right)+\alpha u_{t-1}+\varepsilon_{t}
$$

describe la variación de la variable $y_{t}$ alrededor de su tendencia a largo plazo en términos de factores exógenos, $x$, integrados de orden cero y la corrección del error $\alpha u_{t-1}=\alpha\left(y_{t-1}-\beta_{1}-\beta_{2} x_{t-1}\right)$, error del equilibrio en el modelo de cointegración (Engle y Granger, 1987).

El análisis de la estacionariedad de cada una de las series con objeto de evitar relaciones espúreas, la determinación de posibles relaciones de cointegración mediante el enfoque de máxima verosimilitud de Johansen (1988 y 1992) y la estimación del proceso dinámico de ajuste a la relación de equilibrio a largo plazo mediante un MCE, constituye la pauta para seguir.

Una aproximación econométrica a la cuestión debe combinar el conocimiento del fenómeno, el pensamiento de otros autores y relacionar claramente los efectos con sus causas (Amemiya, 1980). Destacando el protagonismo de la especificación en la elaboración de un modelo econométrico que analice la relación causal entre el comportamiento demográfico y el ciclo inmobiliario el análisis de cointegración complementa el enfoque. Su aplicación de forma flexible contribuye a mejorar la especificación del modelo causal. El análisis de cointegración permite aproximar el conocimiento real del fenómeno económico en etapas avanzadas de su análisis.

En el caso que nos ocupa una aproximación a la relación descrita se realiza desarrollando un ejercicio empírico que intenta capturar las relaciones temporales causales entre el ciclo inmobiliario y el fenómeno demográfico de forma independiente y agregada como una primera aproximación a la cuestión. Contrastar la existencia o no de una relación de equilibrio a largo plazo entre ambas variables, el cáracter de exogeneidad o endogeneidad de los procesos dentro de la relación de equilibrio a largo plazo y el análisis de la interacción dinámica constituyen los objetivos fundamentales del estudio.

\section{Análisis empírico}

El modelo que intenta capturar las relaciones temporales causales entre la dinámica del ciclo inmobiliario y el fenómeno demográfico se verá condicionado por 
las propiedades estadísticas de la información. En este ejercicio se han tomado los datos de VIVIENDAS y NACIMIENTOS elaborados por el Ministerio de Fomento e Instituto Nacional de Estadística (INE) para el periodo comprendido entre los meses de enero de 1975 y marzo de 2013. La muestra está integrada por 459 observaciones de frecuencia mensual. El período de análisis incluye cambios relevantes en la dinámica de ambas variables. La evolución de la natalidad constituye un fenómeno determinante en el desarrollo demográfico español durante las últimas décadas. El descenso registrado de forma continua e intensa por los indicadores desde la segunda mitad de la década de los setenta del siglo XX ha hecho que España haya pasado de tener una de las tasas de natalidad más elevadas de Europa durante la década anterior a una situación diferente que condiciona además el desarrollo demográfico posterior. Desde 1997 hasta 2007, el mercado de la vivienda español se caracterizó por un claro comportamiento expansivo. En la década de los ochenta del pasado siglo había experimentado también una burbuja inmobiliaria desinflada con la ralentización de la actividad económica en el inicio de los noventa (Martínez y García, 2014).

De la evolución temporal de las series se desprende, a priori, un comportamiento no estacionario (figural).

Como fenómeno demográfico la natalidad analiza el total de nacimientos acaecidos en el seno de una población en su conjunto, y genera la incorporación de efectivos poblacionales al marco demográfico de referencia mediante el nacimiento como suceso característico, y junto a la mortalidad y fenómeno migratorio integra el comportamiento demográfico. Como aproximación al ciclo inmobiliario las viviendas iniciadas, VIVIENDAS, permiten visualizar la dinámica del mercado de la vivienda.

Formalmente se contrasta la hipótesis de estacionariedad siguiendo las pautas tradicionales de los estudios con series temporales. La existencia de raíces unitarias dirige el análisis al uso de vectores autorregresivos (VAR). El contraste de estacionariedad se realiza mediante test de Dickey-Fuller Aumentadas (ADF), y se comprueba que ambas variables son no estacionarias e integradas de primer orden. La hipótesis nula a contrastar existencia de raíces unitarias, no es rechazada en niveles pero sí en primeras diferencias en ambos casos (tabla 1).

La interdependencia entre ambas series consideradas inicialmente endógenas mediante la estimación de un VAR visualiza cada variable como una función del pasado de ambas. A partir de la determinación del retardo óptimo ${ }^{4}$ se estima un VAR para ambos procesos con una amplitud temporal de 13 retardos utilizando solo los estadísticamente significativos 1-4 y 12-13, como resultado de la aplicación de pruebas de máxima verosimilitud ${ }^{5}$.

4 Se utilizan como criterios de información razón de verosimilitud (LR), error de predicción final (FPE), Akaike (AIC), Schwartz (SB) y Hannan-Quinn (HQ)

5 VAR estimado después de analizar y corregir el comportamiento de los residuos mediante la introducción de variables dummy como factores exógenos al sistema. 
Figura 1. Evolución temporal de las variables NACIMIENTOS y VIVIENDA

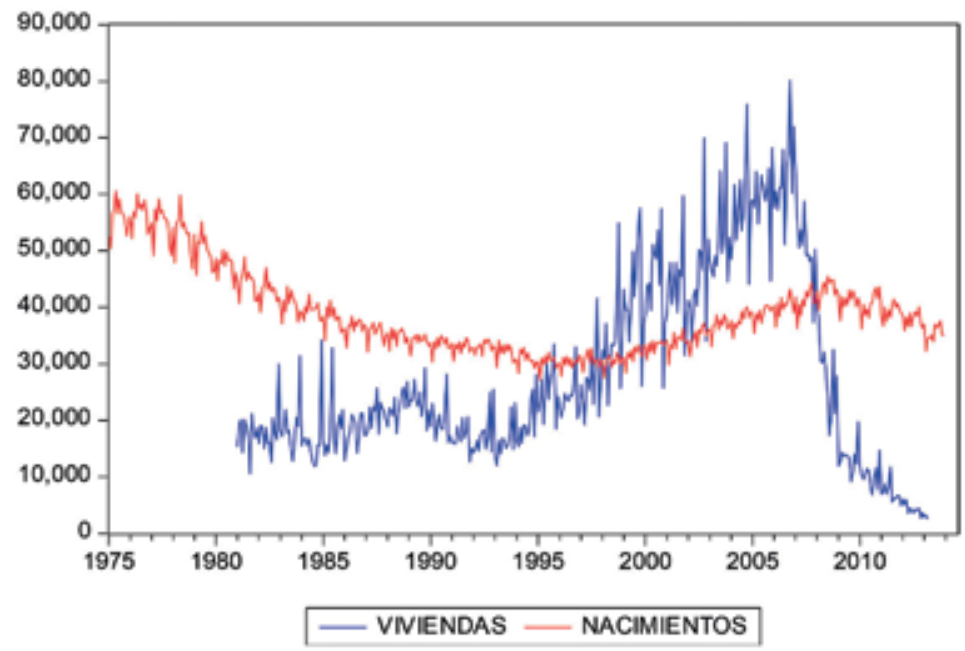

Fuente: Instituto Nacional de Estadística. Ministerio de Vivienda.

Al ser ambas series no estacionarias, series de memoria larga comprobar si están o no cointegradas resulta obligado. Como etapa previa la aplicación del test de causalidad de Granger permite evaluar la endogeneidad entre ambos procesos. La aplicación de la prueba en bloque revela la existencia de una relación de causalidad bidireccional $\left(\chi_{\text {VIVIENDAS-NACIMIENTOS }}^{*}=30,6285 ; \chi_{\text {NACIMIENTOS-VIVIENDAS }}^{*}=65,0413\right)$ además de aproximar qué parte de los valores actuales de una variable se pueden explicar a partir de los valores corrientes y retardados de la otra. Del análisis de hasta 50 retardos entre ambas series se comprueba la existencia de una relación causal bidireccional en sentido Granger entre los retardos 4 y 25. A partir de dicha referencia temporal la causalidad de la variable demográfica perdería significatividad estadística pero no la serie económica (figura 2).

Tabla 1. Análisis de cointegración. 1975.01-2013.03

\begin{tabular}{|c|c|c|c|c|c|c|}
\hline \multicolumn{7}{|c|}{ Test de raíces unitarias } \\
\hline \multicolumn{7}{|c|}{ Augmented Dickey-Fuller test statistic (ADF) } \\
\hline Variable & & Levels & p-value & \multicolumn{2}{|c|}{ First differences } & p-value \\
\hline Nacimientos & & $-1,853338$ & 0,0609 & \multicolumn{2}{|c|}{$-2,752132$} & 0,0059 \\
\hline Viviendas & & $-0,967626$ & 0,2977 & \multicolumn{2}{|c|}{$-3,598825$} & 0,0003 \\
\hline \multicolumn{7}{|c|}{ Determinación del retardo óptimo } \\
\hline Lag & $\log \mathrm{L}$ & LR & FPE & AIC & SC & HQ \\
\hline 13 & $-6672,920$ & $101,2148^{*}$ & $1,92 \mathrm{e}+13$ & 36,26372 & $36,83374 *$ & $36,49011^{*}$ \\
\hline 14 & $-6669,891$ & 5,584813 & $1,94 \mathrm{e}+13$ & 36,26896 & 36,88119 & 36,51212 \\
\hline
\end{tabular}




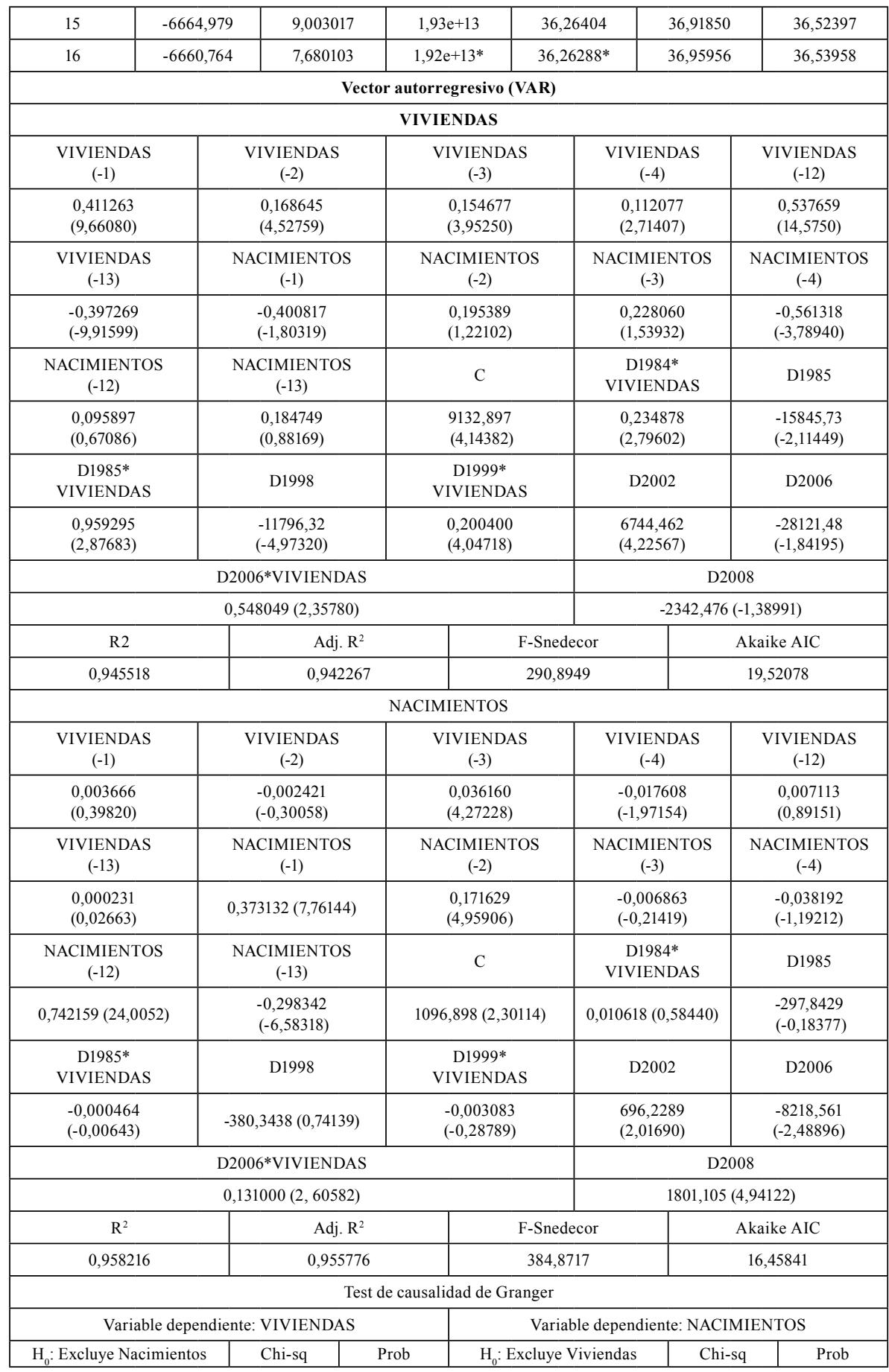




\begin{tabular}{|c|c|c|c|c|c|}
\hline & 30,6285 & 0,0000 & 65,0413 & 0,0000 \\
\hline \multicolumn{5}{|c|}{ Test de cointegración de Johansen } \\
\hline \multicolumn{7}{|c|}{ Trend assumption: Linear deterministic trend } & \multicolumn{2}{|c|}{ Lags interval (in first differences): 1 to 4, 12 to 13} \\
\hline Hypothesized No. of CE(s) & Eigenvalue & Trace Statistic & 0,05 Critical Value & Prob.** \\
\hline None * & 0,052533 & 24,18035 & 15,49471 & 0,0019 \\
\hline At most 1 & 0,010804 & 4,051918 & 3,841466 & 0,0441 \\
\hline
\end{tabular}

Fuente: Elaboración propia.

Figura 2. Causalidad en sentido Granger

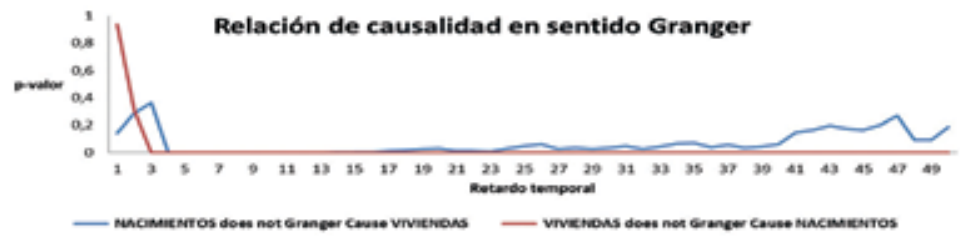

Fuente: Elaboración propia.

Mediante el test de Johansen se detecta la existencia de una relación de cointegración entre ambos procesos sin tendencia determinista y con intercepto. La prueba no rechaza la hipótesis nula de existencia de al menos una relación de cointegración según la prueba de la traza y del máximo valor propio para un nivel de significatividad del uno por ciento. Con objeto de ajustar el modelo propuesto y contemplar la dinámica de ajuste de las variables, tanto a corto como a largo plazo, se especifica un MCE destacando como dato a analizar la velocidad de ajuste hacia el equilibrio.

Cuando dos variables están cointegradas es posible diferenciar entre una relación de largo y corto plazo. Mediante una combinación lineal dos series, VIVIENDAS y NACIMIENTOS, ambas de memoria larga, se transforman en una nueva variable de memoria corta (tabla 2). A partir de la ecuación de cointegración estimada

VIVIENDAS(-1)+4,774702 NACIMIENTOS(-1)-200210,0

se comprueba el dominio y significatividad estadística para el ciclo demográfico en la determinación de la trayectoria del ciclo inmobiliario del efecto a largo plazo 4,774702( $\left.\mathrm{t}^{*}=3,37576\right)$ en relación con el corto plazo $-0,043209\left(\mathrm{t}^{*}\right.$ 0,19901). Las regresiones que aproximan ambos procesos son estadísticamente significativas ${ }^{6}$ si bien presenta una bondad ligeramente superior la modelización de la serie demográfica $\left(\bar{R}^{2}=0,7703\right)$ en relación con la de vivienda $\left(\bar{R}^{2}=0,7153\right)$ y la distribución de los residuos satisface los supuestos de normalidad, independencia serial y homoscedasticidad.

6 Dado que la técnica está afectada por el carácter endógeno de las variables, el análisis de los coeficientes de regresión estimados, su significatividad estadística, la estimación del coeficiente de determinación ajustado, entre otros contrastes, resulta insuficiente para evaluar los resultados obtenidos. 
El tamaño y significatividad estadística del término de corrección de error recoge la velocidad de ajuste al equilibrio que solo es estadísticamente significativa para el ciclo inmobiliario $-0,039384\left(t^{*}=-4,2627\right)$. La estimación obtenida visualiza un proceso lento en la restauración del equilibrio cuando se producen desajustes en la relación de largo plazo. Economistas y económetras identifican el término equilibrio con connotaciones diferentes. Para los primeros el equilibrio describe una situación de igualdad entre los registros actuales y los deseados. Para los segundos cualquier relación de largo plazo entre variables no estacionarias denotaría un escenario de equilibrio. Desde dicha perspectiva el equilibrio econométrico se configura como un objetivo más realista y alcanzable dado que no requiere expresamente la actuación de fuerzas de mercado o acuerdos entre agentes económicos. En el análisis realizado se comprueba que en el largo plazo es la serie económica la que de forma lenta restaura la condición de equilibrio alterada en el corto plazo.

Un resultado importante de cara a la investigación teórica se deriva del análisis de la causalidad. A corto plazo el ciclo inmobiliario presenta un claro nivel de endogeneidad. La evolución de la variable económica se explica fundamentalmente a partir de su propia dinámica y de la influencia derivada de las variables dummy que con una especificación aditi ${ }^{*}$ en 1985 ( $\left.\mathrm{t}^{*}=-2,4912\right), 1998$ ( $\left.\mathrm{t}^{*}=-4,73714\right) \mathrm{y}$ $2002\left(\mathrm{t}^{*}=4,7636\right)$, respectivamente y multiplicativa en $1984\left(\mathrm{t}^{*}=2,90173\right), 1985$ $\left(\mathrm{t}^{*}=3,11439\right), 1999\left(\mathrm{t}^{*}=3,76592\right)$ y $2006\left(\mathrm{t}^{*}=2,43590\right)$ justifican cambios estructurales en la dinámica inmobiliaria. La influencia, estadísticamente significativa del factor demográfico se percibe solo en el tercer retardo. En la evolución del fenómeno demográfico su propia dinámica explica fundamentalmente la trayectoria. Se comprueba la significatividad estadística de las variables dummy correspondientes a los años 2002, 2006 y 2008 incorporadas aditivamente y la interacción en el 2006 con el ciclo inmobiliario.

Tabla 2. Estimación modelo de corrección de error

\begin{tabular}{ccc}
\hline \multicolumn{2}{c}{ Vector Error Correction Estimates Included observations:373 t-statistics in [ ] } \\
CointegratingEq: & CointEq1 & \\
\hline VIVIENDAS(-1) & 1.000000 & \\
NACIMIENTOS(-1) & $4.774702[3.37576]$ & \\
\hline C & -200210.0 & D(NACIMIENTOS) \\
\hline Error Correction: & D(VIVIENDAS) & $-0,003176[-1,45400]$ \\
CointEq1 & $-0,039384[-4,26272]$ & $0,002861[0,25576]$ \\
D(VIVIENDAS(-1)) & $-0,586570[-12,3981]$ & $-0,008657[-0,76279]$ \\
D(VIVIENDAS(-2)) & $-0,359236[-7,48414]$ & $0,019124[1,71357]$ \\
D(VIVIENDAS(-3)) & $-0,209067[-4,42940]$ & $-0,016095[-1,66423]$ \\
D(VIVIENDAS(-4)) & $-0,098332[-2,40404]$ & $0,006917[0,79670]$ \\
D(VIVIENDAS(-12)) & $0,527697[14,3711]$ & $0,002013[0,19449]$ \\
D(VIVIENDAS(-13)) & $0,125233[2,86082]$ &
\end{tabular}




\begin{tabular}{|c|c|c|c|}
\hline \multicolumn{2}{|c|}{ D(NACIMIENTOS(-1)) } & $-0,043209[-0,19901]$ & $-0,432405[-8,42283]$ \\
\hline \multicolumn{2}{|c|}{ D(NACIMIENTOS(-2)) } & $0,256447[1,48450]$ & $-0,049830[-1,21993]$ \\
\hline \multicolumn{2}{|c|}{ D(NACIMIENTOS(-3)) } & $0,541921[3,11208]$ & $-0,043949[-1,06740]$ \\
\hline \multicolumn{2}{|c|}{ D(NACIMIENTOS(-4)) } & $0,038634[0,25602]$ & $-0,025350[-0,71049]$ \\
\hline \multicolumn{2}{|c|}{ D(NACIMIENTOS(-12)) } & $0,055460[0,38127]$ & $0,698866[20,3196]$ \\
\hline \multicolumn{2}{|c|}{ D(NACIMIENTOS(-13)) } & $0,162944[0,78667]$ & $0,250654[5,11792]$ \\
\hline \multicolumn{2}{|l|}{$\mathrm{C}$} & $-459,3068[-2,04645]$ & $-51,26854[-0,96608]$ \\
\hline \multicolumn{2}{|c|}{ D1984*VIVIENDAS } & $0,240358[2,90173]$ & $-0,007135[-0,36432]$ \\
\hline \multicolumn{2}{|l|}{ D1985 } & $-18706,93[-2,49212]$ & $-642,0795[-0,36176]$ \\
\hline \multicolumn{2}{|c|}{ D1985*VIVIENDAS } & $1,039065[3,11439]$ & $0,011011[0,13958]$ \\
\hline \multicolumn{2}{|l|}{ D1998 } & $-11260,89[-4,73714]$ & $-43,76638[-0,07787]$ \\
\hline \multicolumn{2}{|c|}{ D1999*VIVIENDAS } & $0,185318[3,76592]$ & $0,000111[0,00956]$ \\
\hline \multicolumn{2}{|l|}{ D2002 } & $7535,577[4,76369]$ & $817,7229[2,18623]$ \\
\hline \multicolumn{2}{|l|}{ D2006 } & $-29193,09[-1,91871]$ & $-9841,444[-2,73559]$ \\
\hline \multicolumn{2}{|c|}{ D2006*VIVIENDAS } & $0,564400[2,43590]$ & $0,157640[2,87741]$ \\
\hline \multicolumn{2}{|l|}{ D2008 } & $-888,6785[-0,60990]$ & $773,2023[2,24424]$ \\
\hline \multicolumn{2}{|l|}{ R-squared } & 0,732165 & 0,783913 \\
\hline Adj, R-squared & & 0,715329 & 0,770331 \\
\hline F-statistic & & 43,48971 & 57,71455 \\
\hline Akaike AIC & & 19,51821 & 16,63416 \\
\hline Akaikeinformationcriteri & & & 36,15649 \\
\hline & Jarque-Bera & Prob & \\
\hline 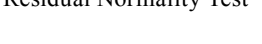 & 18,80334 & & 0,0269 \\
\hline Residual & White & Prob & \\
\hline Heteroskedasticity Test & 153,4767 & & 0,0133 \\
\hline Residual Correlation & LM order 13 & Prob & \\
\hline Test & 2,987361 & & 0,5599 \\
\hline
\end{tabular}

Fuente: Elaboración propia.

El análisis impulso-respuesta (FIR) permite aproximar el efecto de un shock, como permanente o transitorio, sobre el comportamiento de las variables, visualizando dichas relaciones en tiempo, dirección, duración e intensidad (figura 3). Ante una alteración en la dinámica del ciclo inmobiliario la respuesta de ambos procesos es claramente visible en el corto plazo, si bien a largo plazo se percibe un efecto transitorio y ajuste rápido sobre el ciclo inmobiliario y permanente sobre el demográfico. Cuando el shock aleatorio se produce en la variable demográfica esta experimenta una sensible reacción positiva de comportamiento cíclico con 
periodicidad anual. Inicialmente se produce una caída rápida sobre su propia trayectoria que tiende a estabilizarse en un nivel superior en unidades temporales próximas. En el análisis de la FIR los óptimos locales se localizan en las unidades temporales 13 y 25 , respectivamente, visualizando un efecto transitorio con ajuste rápido. El efecto sobre la variable inmobiliaria generaría un ajuste lento con ganancias y pérdidas iniciales tendentes a estabilizarse en el nivel de partida que a largo plazo generaría un efecto de caída permanente.

Figura 3. Función impulso-respuesta

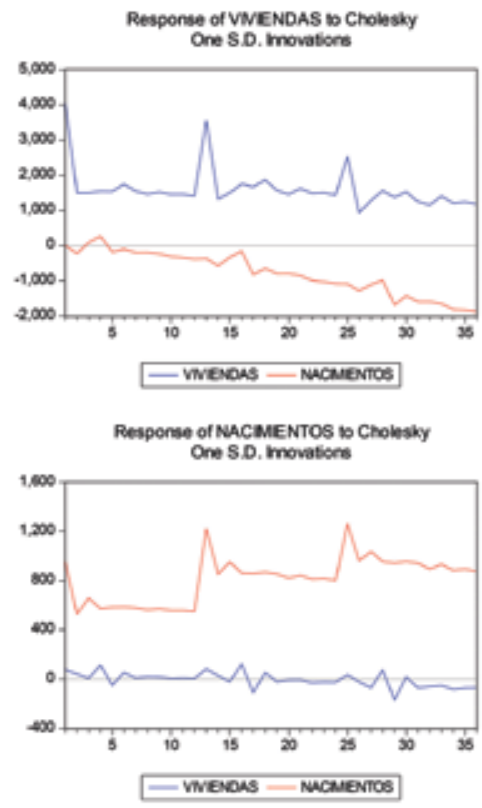

Fuente: Elaboración propia.

El análisis de la descomposición de la varianza profundiza los resultados obtenidos al visualizar el peso de cada variable en la determinación de la desviación típica del error de predicción (figura 4). Se comprueba que a lo largo del horizonte temporal seleccionado en la determinación de la desviación típica del error de predicción de la variable económica y demográfica el peso de la demográfica y económica, respectivamente, es poco significativo. El factor demográfico de forma lenta pero constante participa del registro del indicador del ciclo inmobiliario. A partir de la unidad temporal 15 el peso se cifra de forma creciente en valores superiores a 2 por ciento, 10 por ciento (unidad temporal 26), 15 por ciento (unidad temporal 30 ) y 25 (unidad temporal 36), respectivamente. 
Figura 4. Descomposición de la varianza
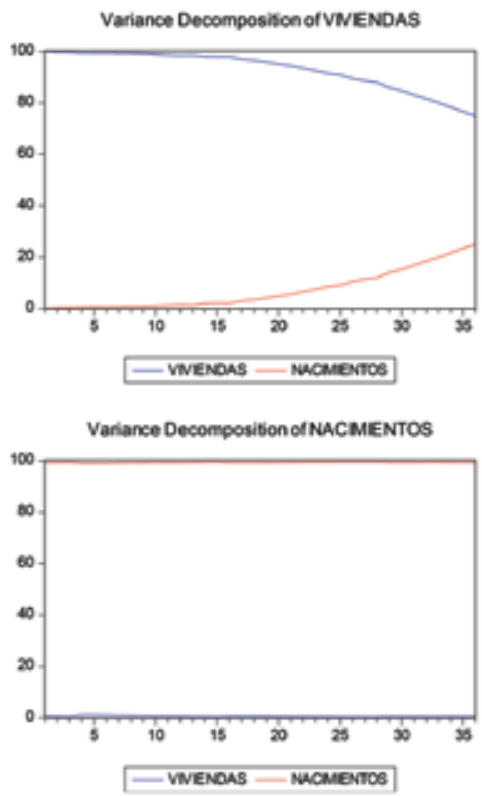

Fuente: Elaboración propia.

La vinculación entre ambos procesos se refrenda al comprobar un peso creciente del ciclo demográfico sobre el inmobiliario. Un 0,2606 por ciento de la reacción del ciclo inmobiliario se explica por la influencia de la variable demográfica aumentando con rapidez hasta el 1,0330 por ciento (unidad temporal 10) y 25,0506 por ciento al final del intervalo temporal considerado. Del análisis del peso de la variable económica sobre el ciclo demográfico se desprende cierta exogeneidad de esta última.

Una vez obtenida la estimación consistente ${ }^{7}$ del MCE las ecuaciones dinámicas permiten aproximar el comportamiento futuro mediante la estimación por MCO de cada proceso individualmente en función de las variables relevantes (tabla 3). En los resultados obtenidos, en línea con las pruebas anteriores, se obtiene para el ciclo inmobiliario y demográfico una estimación estadísticamente significativa $\mathrm{F}^{*}=63,29483$ y $\mathrm{F}^{*}=166,1653$, respectivamente, con capacidad predictiva para la primera (Covariance Proportion=0,805512). Relación de cointegración, dinámica inmobiliaria, factor demográfico y coyuntura económica constituyen los factores explicativos de la variable inmobiliaria con claro dominio de su propia trayectoria

7 La estacionariedad de los residuos de la relación de cointegración se comprueba mediante el test de PhilipsPerron. 
de acuerdo con los coeficientes estandarizados obtenidos. En la trayectoria de la variable demográfica su propia dinámica explica fundamentalmente su evolución.

Tabla 3. Predicción con el modelo de corrección de error

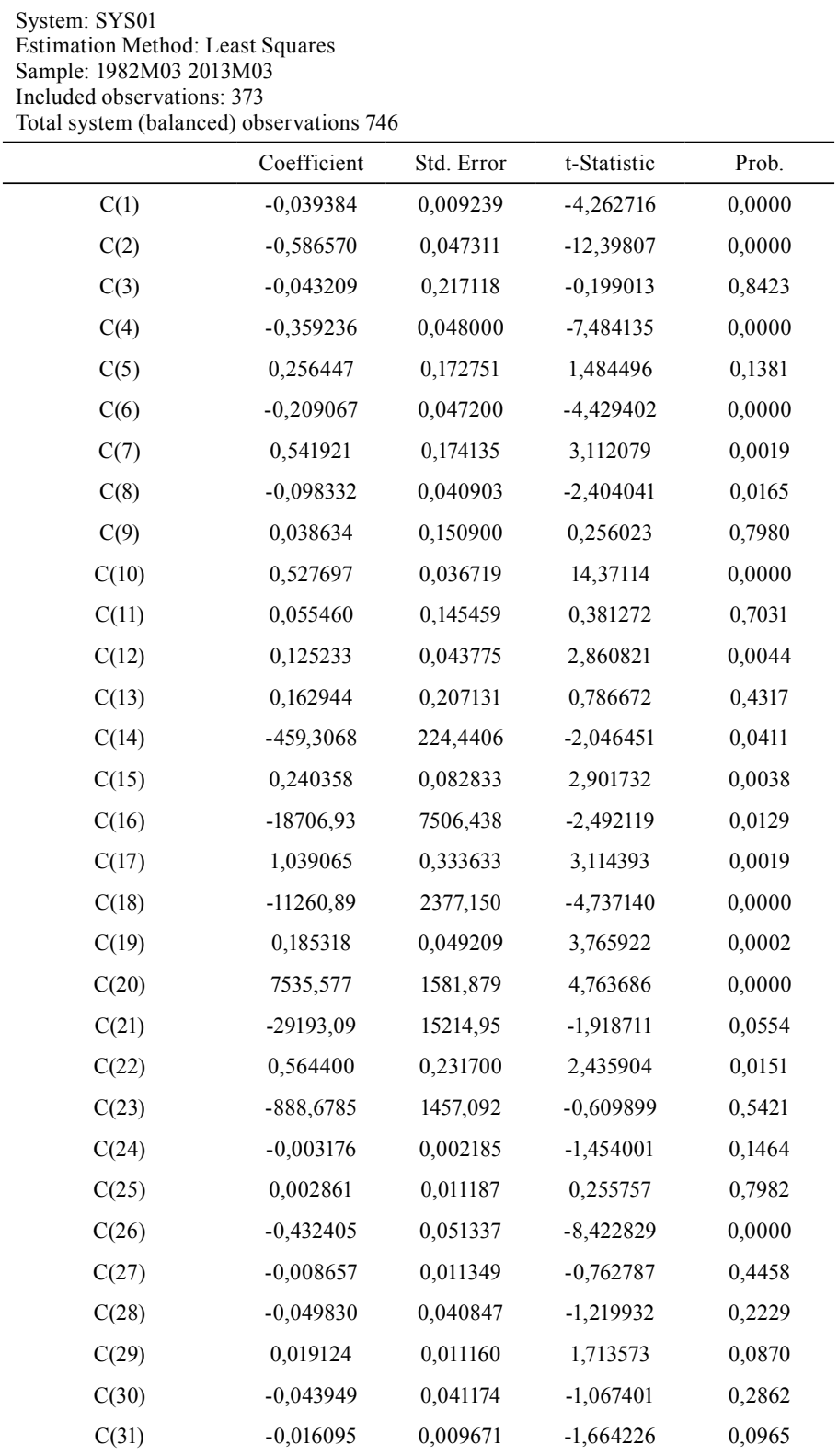


Ciclo inmobiliario y demográfico. Un análisis de cointegración para el caso español

\begin{tabular}{|c|c|c|c|c|}
\hline $\mathrm{C}(32)$ & $-0,025350$ & 0,035680 & $-0,710489$ & 0,4776 \\
\hline $\mathrm{C}(33)$ & 0,006917 & 0,008682 & 0,796703 & 0,4259 \\
\hline $\mathrm{C}(34)$ & 0,698866 & 0,034394 & 20,31959 & 0,0000 \\
\hline $\mathrm{C}(35)$ & 0,002013 & 0,010351 & 0,194494 & 0,8458 \\
\hline $\mathrm{C}(36)$ & 0,250654 & 0,048976 & 5,117916 & 0,0000 \\
\hline $\mathrm{C}(37)$ & $-51,26854$ & 53,06869 & $-0,966079$ & 0,3343 \\
\hline $\mathrm{C}(38)$ & $-0,007135$ & 0,019586 & $-0,364317$ & 0,7157 \\
\hline $\mathrm{C}(39)$ & $-642,0795$ & 1774,887 & $-0,361758$ & 0,7176 \\
\hline $\mathrm{C}(40)$ & 0,011011 & 0,078887 & 0,139575 & 0,8890 \\
\hline $\mathrm{C}(41)$ & $-43,76638$ & 562,0739 & $-0,077866$ & 0,9380 \\
\hline $\mathrm{C}(42)$ & 0,000111 & 0,011635 & 0,009558 & 0,9924 \\
\hline $\mathrm{C}(43)$ & 817,7229 & 374,0332 & 2,186231 & 0,0291 \\
\hline $\mathrm{C}(44)$ & $-9841,444$ & 3597,554 & $-2,735593$ & 0,0064 \\
\hline $\mathrm{C}(45)$ & 0,157640 & 0,054785 & 2,877409 & 0,0041 \\
\hline $\mathrm{C}(46)$ & 773,2023 & 344,5275 & 2,244240 & 0,0251 \\
\hline \multicolumn{5}{|c|}{ 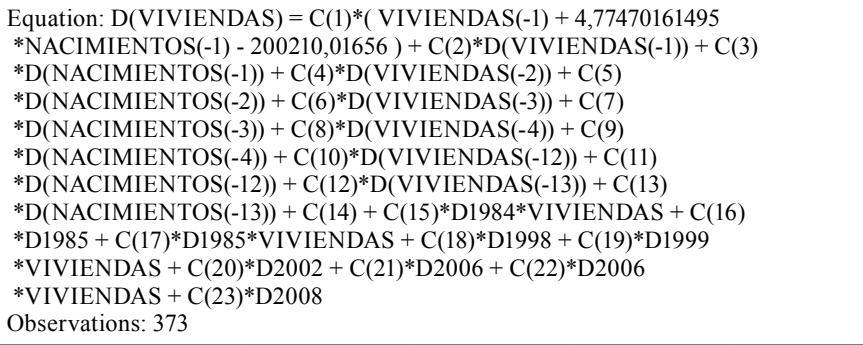 } \\
\hline R-squared & 0,732165 & Mean deper & var & $-44,16086$ \\
\hline Adjusted R-squared & 0,715329 & S,D, depenc & & 7620,063 \\
\hline S.E. of regression & 4065,649 & Sum square & & $5,79 \mathrm{E}+09$ \\
\hline Durbin-Watson stat & 1,941518 & & & \\
\hline \multicolumn{5}{|c|}{ 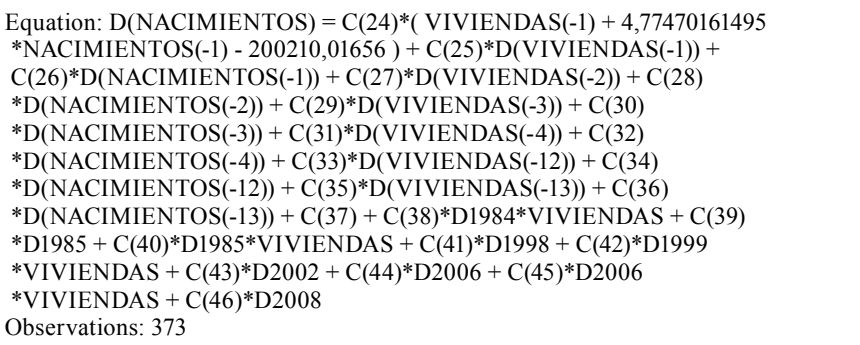 } \\
\hline R-squared & 0,783913 & Mean deper & tvar & $-12,29759$ \\
\hline Adjusted R-squared & 0,770331 & $\mathrm{~S}, \mathrm{D}$, depen & & 2005,927 \\
\hline S.E. of regression & 961,3171 & Sum square & & $3,23 \mathrm{E}+08$ \\
\hline Durbin-Watson stat & 1,998730 & & & \\
\hline
\end{tabular}


Dependent Variable: D(VIVIENDAS)

Method: Least Squares

Sample (adjusted): 1982M03 2013M03

Included observations: 373 after adjustments

\begin{tabular}{|c|c|c|c|c|}
\hline Variable & Coefficient & Std. Error & t-Statistic & Prob. \\
\hline $\mathrm{C}$ & $-498,1858$ & 220,8273 & $-2,255997$ & 0,0247 \\
\hline $\begin{array}{l}\text { VIVIENDAS(-1)+4,77470161495 } \\
\text { *NACIMIENTOS(-1)-200210,01656 }\end{array}$ & $-0,039021$ & 0,008598 & $-4,538204$ & 0,0000 \\
\hline D(VIVIENDAS(-1)) & $-0,581770$ & 0,045570 & $-12,76643$ & 0,0000 \\
\hline D(VIVIENDAS(-2)) & $-0,348654$ & 0,046341 & $-7,523677$ & 0,0000 \\
\hline D(VIVIENDAS(-3)) & $-0,208064$ & 0,045683 & $-4,554548$ & 0,0000 \\
\hline D(NACIMIENTOS(-3)) & 0,389244 & 0,112364 & 3,464143 & 0,0006 \\
\hline D(VIVIENDAS(-4)) & $-0,105008$ & 0,035871 & $-2,927343$ & 0,0036 \\
\hline D(VIVIENDAS(-12)) & 0,544624 & 0,034055 & 15,99271 & 0,0000 \\
\hline D(VIVIENDAS(-13)) & 0,136763 & 0,042927 & 3,185921 & 0,0016 \\
\hline D1984*VIVIENDAS & 0,233619 & 0,082402 & 2,835128 & 0,0048 \\
\hline D1985 & $-18773,46$ & 7483,922 & $-2,508506$ & 0,0126 \\
\hline D1985*VIVIENDAS & 1,053580 & 0,332536 & 3,168322 & 0,0017 \\
\hline D1998 & $-11134,65$ & 2365,299 & $-4,707501$ & 0,0000 \\
\hline D1999*VIVIENDAS & 0,183314 & 0,049157 & 3,729180 & 0,0002 \\
\hline D2002 & 7651,028 & 1574,067 & 4,860674 & 0,0000 \\
\hline D2006*VIVIENDAS & 0,122615 & 0,023493 & 5,219135 & 0,0000 \\
\hline R-squared & 0,726735 & \multicolumn{2}{|c|}{ Mean dependent var } & $-44,16086$ \\
\hline Adjusted R-squared & 0,715253 & \multicolumn{2}{|c|}{ S.D. dependent var } & 7620,063 \\
\hline S.E. of regression & 4066,195 & \multicolumn{2}{|c|}{ Akaike info criterion } & 19,50075 \\
\hline Sum squared resid & $5,90 \mathrm{E}+09$ & \multicolumn{2}{|c|}{ Schwarz criterion } & 19,66897 \\
\hline Log likelihood & $-3620,890$ & \multicolumn{2}{|c|}{ Hannan-Quinn criter. } & 19,56755 \\
\hline F-statistic & 63,29483 & \multicolumn{2}{|c|}{ Durbin-Watson stat } & 1,938314 \\
\hline Prob(F-statistic) & 0,000000 & & & \\
\hline
\end{tabular}
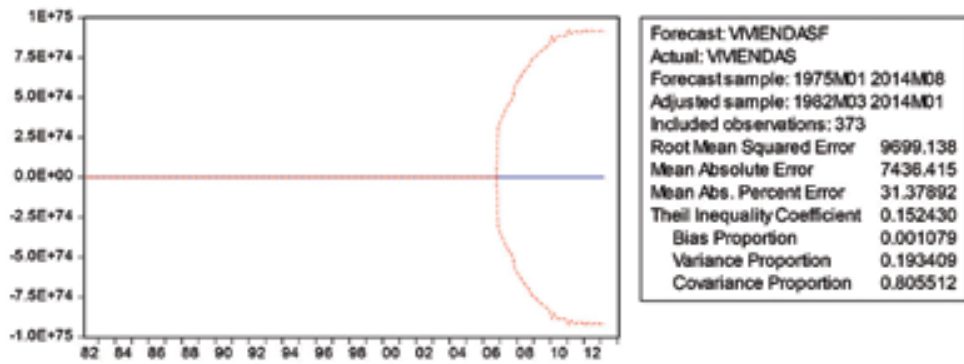


\begin{tabular}{|c|c|c|c|c|}
\hline \multicolumn{5}{|c|}{$\begin{array}{l}\text { Dependent Variable: D(NACIMIENTOS) } \\
\text { Method: LeastSquares } \\
\text { Sample (adjusted): } 1981 \mathrm{M} 01 \text { 2013M03 } \\
\text { Includedobservations: } 387 \text { afteradjustments }\end{array}$} \\
\hline Variable & Coefficient & Std. Error & t-Statistic & Prob. \\
\hline D(NACIMIENTOS(-1)) & $-0,428039$ & 0,045644 & $-9,377702$ & 0,0000 \\
\hline D(NACIMIENTOS(-12)) & 0,771160 & 0,031434 & 24,53235 & 0,0000 \\
\hline D(NACIMIENTOS(-13)) & 0,272954 & 0,048539 & 5,623420 & 0,0000 \\
\hline D2002 & 654,1263 & 384,2212 & 1,702473 & 0,0895 \\
\hline D2006 & 108,4572 & 359,6409 & 0,301571 & 0,7631 \\
\hline D1999*VIVIENDAS & 0,000686 & 0,010613 & 0,064620 & 0,9485 \\
\hline D2008 & 558,1596 & 338,1621 & 1,650568 & 0,0997 \\
\hline R-squared & 0,753722 & Mean dependentvar & & $-29,94315$ \\
\hline Adjusted R-squared & 0,749834 & S.D. dependentvar & & 2026,846 \\
\hline S.E. of regression & 1013,760 & Akaikeinfocriterion & & 16,69864 \\
\hline Sum squaredresid & $3,91 \mathrm{E}+08$ & Schwarzcriterion & & 16,77024 \\
\hline Log likelihood & $-3224,187$ & Hannan-Quinncriter. & & 16,72703 \\
\hline Durbin-Watson stat & 2,061833 & & & \\
\hline
\end{tabular}

Fuente: Elaboración propia.

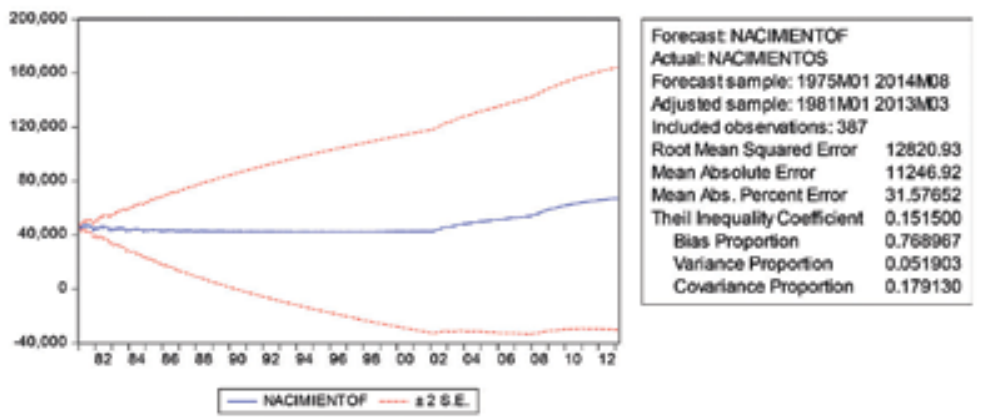

\section{Conclusiones}

En este trabajo se investiga la relación existente entre el ciclo inmobiliario (VIVIENDAS) y el demográfico (NACIMIENTOS) en España durante el período comprendido entre 1975 y 2013 con periodicidad mensual. Destaca la oportunidad del enfoque al tratarse de una relación causal analizada en la literatura económica, constituyendo una de las principales novedades del mismo la utilización de series temporales homogéneas y la contrastación de la interacción entre el corto y largo plazo. Se analiza, en concreto, la existencia de relaciones de equilibrio a largo plazo en un entorno bivariante contrastando el carácter endógeno de ambas variables a través del análisis de un MCE que integra las relaciones de corto y largo plazo, 
detectadas mediante un análisis de cointegración previo (Díaz y Llorente, 2012). Las diferentes pruebas realizadas confirman la validez del modelo estimado.

El alcance de la relación causal en horizontes temporales de corto y largo plazo no siempre se ha utilizado conscientemente en la toma de decisiones. El conocimiento del comportamiento demográfico de un ámbito territorial permite disponer de una herramienta básica y adelantada para la planificación urbana territorial y socio-económica (Mankiw y Weil, 1989; Poterba, 1991). Con carácter general los argumentos demográficos han servido de poco a la hora de influir en las políticas socioeconómicas, puesto que mientras que los comportamientos demográficos generan efectos en el largo plazo las actuaciones políticas suelen estar necesitadas de visibilidad en el corto plazo.

Del análisis de las características que definen la vivienda como bien de naturaleza económica (necesario, importante, duradero...) se desprende la vinculación de las decisiones de acceso a esta a intervalos temporales de largo plazo. La vivienda acompaña al efectivo poblacional a lo largo de su ciclo de vida. Desde la misma perspectiva el fenómeno demográfico se modeliza como un recurso cuya trayectoria se proyecta en el horizonte temporal de largo plazo (Díaz, Costa y Llorente, 1997).

Los resultados obtenidos confirman:

- La existencia de una relación de equilibrio a largo plazo entre ambas variables. La relación de cointegración obtenida recoge una combinación lineal de variables de memoria larga, serie no estacionaria, en una nueva de memoria corta, estacionaria, que comparten una tendencia estocástica común. La reciprocidad que muestra la ecuación cointegradora entre ambos procesos es inversamente proporcional.

- Relación de causalidad. El análisis de la causalidad en sentido Granger permite aproximar los períodos temporales de causalidad en sentido bidireccional y unidireccional. Se comprueba el carácter endógeno del proceso inmobiliario. En el ajuste de las desviaciones del equilibrio generadas en el corto plazo es la variable inmobiliaria la que responde para recuperar la convergencia en el equilibrio a largo plazo. El resultado obtenido de la aplicación de la prueba de Granger permite visualizar, además, el comportamiento de las pautas de fecundidad, su trayectoria y calendario.

- El dominio del ciclo inmobiliario. La estimación del MCE pone de manifiesto el dominio del ciclo inmobiliario en la relación analizada y la identificación de los factores explicativos. Confirma el dominio de las decisiones a largo plazo comprobando que las correspondientes al corte ni siquiera son estadísticamente significativas. Además de la relación de cointegración, su propia dinámica, el factor demográfico la significatividad de las variables dummy incluidas en la especificación son destacables porque permiten visualizar los cambios estructurales registrados en diferentes referencias temporales de la economía 
española, segunda mitad de la década de los ochenta, final de los noventa e inicio de las primeras señales de la actual en 2002 y 2006. Los resultados obtenidos permiten ubicar en el entorno muestral hechos recientes de la economía española.

- Resultados coherentes con estudios previos. Como una primera aproximación al estudio de la interacción entre ambos ciclos los resultados obtenidos son coherentes con la literatura económica y visualizan hechos reales. En la determinación de la demanda de vivienda se diferencian dos componentes, demanda a corto y largo plazo. Las variables demográficas son determinantes en la trayectoria de largo plazo que además se suele utilizar como una variable adelantada del comportamiento futuro. En el corto plazo son las variables financieras, económicas y fiscales las determinantes, no obstante, los resultados permiten identificar el entorno temporal de su influencia.

\section{Referencias}

Amemiya, T. (1980). Selection of Regressors. International Economic Review, 21(2), 331-354.

Becker, G.S. (1960). A Treatise of the Family. Harvard University Press, Harvard. (Ed. Castellana 1987). Alianza Universidad. Madrid.

Bover, O. (1993, enero). Un modelo empírico de la evolución de los precios de la vivienda en España. Investigaciones Económicas, XVII(1), 65-86.

Clark, W., y Dieleman, F. (1996). Households and housing: Choice and outcomes in the housing market. New Brunswick: Centre for Urban Policy Research.

Climent, F., y Meneu, R. (2003). Relaciones de equilibrio entre demografía y crecimiento económico en España. Estudios sobre la Economía Española, $\mathrm{N}^{\mathrm{o}}$ 163. FEDEA.

Dettling, L. y Kearney, M., 2014. House prices and birth rates: The impact of the real estate market on the decision to have a baby. Journal of Public Economics, 110, 82-100.

Díaz, M., Costa, E., y Llorente, M. (1997, julio). Análisis econométrico de la demanda de vivienda en España. El papel de la financiación externa. Actualidad Financiera, II(7), 133-151.

Díaz, M., y Llorente, M. (2012). Births and housing needs in Spain. An analysis of causality. European Population Conference 2012. Estocolmo, Suecia.

Eichholtz, P., y Lindenthal, T. (2010). Demographics, human capital and the demand for housing. Documento de Trabajo. Maastricht University. 
Engle, R., y Granger, S. (1987, marzo). Co-Integration and Error Correction: Representation, Estimation, and Testing. Econometrica, 55(2), 251-276.

Feijten, P., y Mulder, C. (2002). The timing of household events and housing events in the Netherlands: A longitudinal perspective. Housing Studies, 17, 773-792.

Green, R., y Hendershott, P. (1995). Age, housind demand and real house prices. Regional Science and Urban Economic, No 26, 468-480.

Guisán, C. (2002). Causalidad y cointegración en modelos econométricos: Aplicaciones a los países de la OCDE y limitaciones de los test de cointegración. Working Paper Series Economic Development, N ${ }^{\mathrm{o}}$ 61. Universidad de Santiago de Compostela.

Hamilton, B.W. (1991). The baby boom, the baby hust and the housing market. A second look. Regional Science and Urban Economic, № 21, 547-552.

Johansen, S. (1988). Statistical analysis of cointegration vectors. Journal of Economics Dynamics and Control, $\mathrm{N}^{\circ} 12$.

Johansen, S. (1992). Determination of cointegration rank in the presence of a linear trend. Oxford Bulletin of Economics and Statistics, 54(3), 383-398.

Lim, J., Lee, J. (2013). Demographic changes and housing demands by scenarios with ASFRs. International Journal of Housing Markets and Analysis, 6(3), 317-340.

Mankiw, N.G., Weil, D.N. (1989). The baby boom, the baby just and the housing market. Regional Science and Urban Economic, $\mathrm{N}^{\mathrm{o}}$ 19, 235-258.

Martínez, J., y García, D. (2014). Auge y crisis del sector inmobiliario reciente: interrelación con la política económica. Documento de Trabajo, nº5/2014. Instituto de Estudios Fiscales.

Mulder, C. (2006). Population and housing: A two-sided relationship. Demographic Research, 15(13), 401-412.

Myers, D. (1990). Housing demography: Linking demographic structure and housing markets. Madison, Wisc.: University of Wisconsin Press.

Naredo, J. (2004). Perspectivas de la vivienda. Información Comercial Española, ${ }^{\circ}$ $815,143-154$.

Poterba, J. (1991). House price dynamics: the role of tax policy and demography. Brookings Papers on Economics Activity, 2:1991, 143-203.

Vinuesa, J. (2003). Población y demanda de vivienda: una relación a reconsiderar. La dinámica demográfica protagonista del territorio. Actas del VIII Congreso de la Población Española. Universidad de Santiago de Compostela.

Vinuesa, J. (2005). Dinámica demográfica, mercado de la vivienda y territorio. Papeles de Economía Española, 104, 253-269. 